\title{
Frailty assessment: clinical application in the hospital setting
}

\author{
CW Wong *
}

\section{A B S T R A C T}

Because of their heterogeneous health status, older people's health care needs differ. The concept of frailty, characterised by a decline in physiological reserve with increased vulnerability to stress, is emerging in clinical practice. Characterising older people by frailty could help to predict their prognosis and health outcomes. Further, consideration of frailty in clinical practice could facilitate the determination of patient-centred goals for effective care delivery. In the hospital setting, identification of frailty could guide management plans for older patients upon disposal from emergency departments; risk stratification by frailty allows appropriate decision making about surgical or invasive interventions; selection of frail people to acute geriatric wards for integrated care improves outcomes; end-of-life care for people at advanced stages of frailty improves the quality of their last days of life; and pre-discharge comprehensive geriatric assessment for frail people helps arrangement of post-discharge programmes according to individual needs. Although the emphasis on frailty is growing in clinical practice, there are challenges regarding implementation that need to be addressed.

\section{Hong Kong Med J 2018;24:623-8}

DOI: $10.12809 / \mathrm{hkmj} 187572$

CW Wong *, FHKAM (Medicine), FHKCP

Department of Medicine and Geriatrics, Caritas Medical Centre, Hong Kong

* Corresponding author: wong.chitwai@gmail.com

\section{Introduction}

Age-related cumulative decline in multiple body systems that increases the vulnerability to stress and adverse health outcomes brought about the concept of frailty. Frailty is defined as "a medical syndrome with multiple causes and contributors that is characterised by diminished strength, endurance, and reduced physiologic function that increases an individual's vulnerability for developing increased dependency and/or death". ${ }^{1}$ Although physical frailty has received the most attention, frailty is a multidimensional state that also comprises cognitive and psychosocial components. These components interact with each other and with the external environment to determine the frailty state of an older person in a given setting. Frailty is distinct from comorbidities and disability, despite the fact that they often coexist and are interrelated. ${ }^{2}$ Co-morbidity is the concurrent presence of two or more medical diseases, whereas disability is difficulty performing activities of daily living and indicates dependency. Frail people are likely to have co-morbidities, and frailty may limit their performance of daily activities. Further, co-morbidities increase the likelihood of being frail and disabled, and disability may exacerbate both frailty and co-morbidities. All of these factors add complexity to the incorporation of frailty into clinical practice.

The prevalence of frailty varies among studies and depends on the working definition of frailty. Using a well-known approach, the frailty phenotype model, ${ }^{3}$ a systematic review reported a weighted average prevalence of $9.9 \%$ for frailty and $44.2 \%$ for pre-frailty in community-dwelling older people aged $\geq 65$ years. ${ }^{4}$ One study in Hong Kong based on the same criteria found similar prevalence levels of $7.9 \%$ and $50.6 \%$ for frailty and pre-frailty, respectively. ${ }^{5}$ The prevalence of frailty increases with age, rising markedly from $4 \%$ in people aged 65 to 69 years to $16 \%$ in those aged 80 to 84 years and $26 \%$ in those aged $>85$ years; the prevalence of frailty was also higher in women $(9.6 \%)$ than in men $(5.2 \%){ }^{4}$

There is increasing emphasis of frailty from literature to clinical practice because of its clinical implications. Frailty is associated with a range of adverse outcomes such as falls, disability, hospitalisation, institutionalisation, and death, ${ }^{3}$ which significantly impact both patients and society. Even though the prevalence of frailty among older people has been constant over time, with the ageing of the population, the number of older people with frailty is expected to continuously increase in the future. In turn, this will pose an increasing burden on the health care system. However, detection of frailty allows risk stratification to predict individual outcomes of interventions or medication treatment. This facilitates better-informed decision making for clinicians, patients, and caregivers while 


\section{衰弱評估：醫院內的臨床應用} 王哲慧

由於老年人的健康狀況多樣化, 他們的醫療需求亦各有不同。近年衰 弱的慨念漸漸呈現在臨床實踐中。衰弱起因於生理系統功能衰退, 造 成身體儲備能力降低及對壓力源的抵抗能力下降。識別衰弱症狀有助 預測患者的健康情況。在臨床實踐中, 考慮衰弱在內的因素有助確立 患者為本的治理目標。在醫院內, 偵測出衰弱有助計劃在急症科出院 時的治理方案; 在外科手術及入侵性檢查或治療前, 對患者作出衰弱 風險分層有助醫護人員及病人暸解風險及作出適當決策; 安排衰弱患 者到老人科病房進行周全性的老年醫學評估, 能促進治療效果及使病 患者獲得妥善照顧; 對晚期衰弱患者的臨終冶理, 可改善他們在生命 最後階段的生活質素; 為衰弱患者提供出院前的綜合老年評估, 有助 安排個人化的出院治理方案。儘管衰弱症狀逐漸備受關注, 但在臨床 實踐方面仍存在挑戰。 formulating appropriate management plans. As frailty is a dynamic process and is reversible, ${ }^{6}$ there are targeted interventions to revert frailty, to reduce the level of frailty, or to slow its progression. Overall, consideration of frailty within the health care system is beneficial at both the patient level, as it allows patient-centred and goal-oriented care, and at the societal level, as it facilitates better health care planning and resource allocation in the community. This article focuses on the clinical application of frailty assessment in the hospital setting.

\section{Screening and assessment for frailty}

Despite the conceptual definition of frailty, a working definition is required to identify and quantify frailty in both research and clinical practice. The two most common approaches are the frailty phenotype model and the frailty index of cumulative deficit model. The frailty phenotype model was described using data from the Cardiovascular Health Study. ${ }^{3}$ It defines frailty as the manifestation of three or more of the five physical indicators: unintentional weight loss $(10 \mathrm{lbs}$ in the past year), self-reported exhaustion, weakness (in terms of grip strength), slow walking speed, and low physical activity. People who have one or two of the indicators are pre-frail, while those who have none of the indicators are non-frail. The frailty index of cumulative deficits model was developed using data from the Canadian Study of Health and Aging.? It construes frailty as the accumulation of a variety of health deficits including co-morbidities, physical and cognitive impairment, psychosocial risk factors, and geriatric syndrome. It is a quantitative measure that calculates the proportion of deficits present out of the total number of parameters considered. A higher proportion of deficits present is associated with a higher level of frailty, which is represented on a continuum from fitness to mild, moderate, and severe frailty. The numbers of deficits measured ranged from 92 in the initial list to around 30 to 40 in subsequent studies. ${ }^{7,8}$

The frailty phenotype model and the frailty index require measurement, retrieval of patient clinical data, and calculation, all of which are time-consuming and may be unfeasible in routine practice. A variety of more "user-friendly" and validated methods have emerged for practical use. The simplest ones that require the shortest time are single-item measurements of gait speed or a Timed Up and Go test. ${ }^{9}$ Some measures are derived from the above models, such as the FRAIL scale, which is based on the phenotype model and takes the form of a questionnaire without measurement. ${ }^{10}$ In addition, the Edmonton Frail Scale, which includes multidimensional questions about the patient's general health, functional performance, nutrition, medication use, and social support, in addition to clock drawing and a Timed Up and Go test, is quick to administer. ${ }^{11}$ The Clinical Frailty Scale is based on clinical judgement of an individual's dependency level and health state to describe a nine-point clinical scale with pictographs from very fit (Category 1 ) to terminally ill (Category 9). ${ }^{12,13}$

The choice of instruments depends on the purpose and clinical setting. Gait speed and Timed Up and Go testing are simple for routine assessment. The frailty phenotype model allows detection of prefrailty so that preventive measures can be taken. ${ }^{14}$ The frailty index is better for prediction of long-term mortality. ${ }^{15}$ The FRAIL scale is a screening test that is easy to perform in the community setting. The Edmonton Frail Scale can identify modifiable factors for preoperative optimisation. Nevertheless, upon identification of people with frailty, they should undergo comprehensive geriatric assessment (CGA), which is a multidimensional, interdisciplinary diagnostic process to determine a frail older person's medical, psychological, and functional capacity to develop a coordinated and integrated plan for treatment and long-term follow-up. ${ }^{16}$ Currently, CGA is regarded as the gold standard for detection and management of frailty. ${ }^{17,18}$

\section{Application of the concept of frailty in hospitals}

Older people account for the majority of health care utilisation. According to a Hospital Authority statistical report, patients aged $\geq 65$ years accounted for $53 \%$ of the approximately 8000000 patient-days in hospitals in Hong Kong during 2016 to 2017. ${ }^{19}$ A large proportion of these older patients are expected to have frailty. Accordingly, incorporation of the concept of frailty into care is reasonable. The following subsections illustrate how the concept of frailty facilitates patient management in the hospital setting. 


\section{Emergency department}

Utilisation of hospital care services often begins with emergency department (ED) attendance. Risk stratification of older patients in EDs can facilitate allocation of optimal care to patients with different needs and improve outcomes. Various frailty assessment tools, such as the Clinical Frailty Scale and the frailty index, have been studied in the ED setting to identify older people with frailty and the associated adverse outcomes upon ED discharge. ${ }^{20}$ Studies have shown that compared with those without frailty, patients assessed as frail had a higher risk of revisiting the ED within 6 months of discharge (odds ratio $[\mathrm{OR}]=2.48 ; 95 \%$ confidence interval $[95 \%$ $\mathrm{CI}]=1.25-4.91)^{21}$; a higher risk of hospitalisation, nursing home admission, and higher mortality within 30 days of the index ED visit (hazard ratio $[\mathrm{HR}]=1.98$; $95 \% \mathrm{CI}=1.29-3.05)^{22}$; 16 times the risk of functional decline at 3 month ${ }^{23}$; and significantly higher risk of mortality at 6 months $(\mathrm{HR}=8.68 ; 95 \% \mathrm{CI}=2.6$ 28.94). ${ }^{21}$ In addition, the risk of adverse outcomes increased with higher levels of frailty: $27.4 \%$ of the most frail patients had adverse outcomes within 30 days of ED discharge, compared with $16.2 \%$ of the least frail patients. ${ }^{22}$ Understanding which individual patients have an additional risk of adverse outcomes could facilitate treatment plan formulation upon disposal of patients from EDs-whether the patients need hospital admission or are fit to go home and whether they need further assessment, additional community service, or rehabilitation. For patients in EDs with non-acute conditions that do not require hospital admission, those with frailty might benefit from follow-up community-based care, such as community nursing, community outreach teams, phone follow-up, or referral to geriatric day hospitals for CGA and rehabilitation. For those with less acute conditions that demonstrate an equivocal need for hospital admission, non-frail or less frail patients might be discharged for home treatment and subsequent follow-up, but hospital admission for management may be considered for frail patients.

\section{Surgical and invasive interventions}

Older people with frailty have a limited physiologic reserve to endure surgical operations and are at risk of poorer surgical outcomes. It has been recognised that frailty independently predicts postoperative complications, length of stay, discharge to an institution, and 30-day mortality. ${ }^{24,25}$ This has led to increasing concern about frailty in surgical practice. Accordingly, a recommendation to incorporate the concept of frailty into preoperative assessment has been made. ${ }^{24}$ Frailty has been shown to increase the predictability of conventional risk models for adverse surgical outcomes..$^{25}$ Incorporation of the concept of frailty improves risk stratification and prediction of surgical outcomes to determine who may benefit or be harmed by surgery or whether or not surgery is appropriate or necessary. The anticipated outcomes could facilitate decision making for surgeons and patients and help surgeons to consider less invasive options for those at high operative risk. In addition, frailty assessment enhances preoperative risk management, including medication review, nutritional augmentation, and physiotherapy to minimise postoperative complications. It can also improve postoperative care arrangements, such as close monitoring in an Intensive Care Unit for early complications during the immediate postoperative period, preventive measures against delirium, and attention to hydration, nutrition, and early mobilisation.

\section{Acute geriatric wards}

For older people with frailty who need in-hospital care for their medical conditions, admission to an acute geriatric ward (AGW) is likely to be more advantageous than that to a general medical ward. General medical wards focusing on treatment of medical problems are insufficient to address older people's complex needs. Apart from acute medical problems, interactions with underlying comorbidities and coexisting functional, psychological, and social problems can complicate health outcomes and the independence of older people during and after the acute illness. Acute geriatric wards are designed to provide patient-centred care, including medical care reviews, early rehabilitation, and discharge planning. This is more appropriate for prevention of hospital-related complications such as delirium, falls, pressure sores, and functional decline and for maximisation of recovery, return to the patient's previous level of functioning, and discharging the patient to his/her home in the community. ${ }^{26}$ Central to the operation of AGWs is the application of CGA. In a meta-analysis of 22 randomised controlled trials involving nearly 10315 patients, when compared with patients receiving general medical care, those who received CGA in AGWs were more likely to be alive and in the community at the end of followup (median 12 months) [OR=1.22; 95\% CI=1.11.35], less likely to be institutionalised at the end of follow-up ( $\mathrm{OR}=0.73 ; 95 \% \mathrm{CI}=0.64-0.84)$, and less likely to experience outcomes of death or functional decline $(\mathrm{OR}=0.76 ; 95 \% \mathrm{CI}=0.64-0.9) .{ }^{18}$ Targeting acute geriatric care for frail patients with geriatric syndrome, at perceived risk of institutionalisation and functional or cognitive impairment is even more beneficial than targeting based on age alone. Compared with treatment at a general medical ward, every 13 frail patients admitted to AGW would allow one more patient to be alive and in the community at 1 year $(\mathrm{OR}=1.36$; $95 \% \mathrm{CI}=1.16-1.6)$, whereas ageonly recruitment criteria for AGW admission had no significant benefit. In addition, for frail patients, 
management in an AGW was associated with lower mortality at 3 months $(\mathrm{HR}=0.55 ; 95 \% \mathrm{CI}=0.32$ 0.96), lower risk of an increasing degree of frailty $(\mathrm{OR}=0.23 ; 95 \% \mathrm{CI}=0.13-0.4)$, and lower risk of decline in activities of daily living $(\mathrm{OR}=0.093$; 95\% $\mathrm{CI}=0.052-0.164) \cdot{ }^{27,28}$

\section{End-of-life care}

Frailty is independently associated with mortality. Using the frailty phenotype model, older people with frailty were at increased risk of death compared with a non-frail group over 3 years $(\mathrm{HR}=2.24$; $95 \%$ $\mathrm{CI}=1.51-3.33){ }^{3}$ Worsening of frailty status during the previous 2 years also predicted higher mortality over the next 2 years: non-frail to frail $(\mathrm{HR}=8.1 ; 95 \%$ $\mathrm{CI}=2-32.5)$, pre-frail to frail $(\mathrm{HR}=3.6 ; 95 \% \mathrm{CI}=1.4$ 9.1). ${ }^{29}$ Categorising frailty into different levels allows better characterisation of its correlation with mortality. ${ }^{30}$ In a large-scale longitudinal study of 13717 Chinese people aged $\geq 65$ years, using a frailty index of 39 variables and division into four levels of frailty showed that the mortality rate increased with higher levels of frailty. ${ }^{31}$ The mortality rates at 3 years were $14.4 \%, 28.7 \%, 49.9 \%$, and $73.1 \%$ for those in 1 st quartile (least frail), 2nd quartile, 3rd quartile, and 4th quartile (most frail), respectively. Further, in another study, a 52-item frailty index based on the CGA illustrated that mortality approached $100 \%$ for those with frailty index $\geq 0.5$ by about 20 months. ${ }^{32}$ Frailty measured by the Clinical Frailty Scale has been shown to be an independent predictor of in-hospital mortality. ${ }^{33}$ Following emergency admission of patients aged $\geq 75$ years, in-hospital death increased from around $2 \%$ for those at Clinical Frailty Scale categories 1 to 3 (fit and well) to $24 \%$ for those at category 8 (very severely frail) and $31 \%$ for those at category 9 (terminally ill).

In light of the above, increasing frailty implies that people are at the last phase of life, and it should be considered as an indication for end-of-life care. The prognosis of patients with severe frailty is expected to be even worse when they experience an acute illness or continuous deterioration of a chronic medical illness. In-hospital end-of-life care to avoid futile interventions but promote symptomatic relief, provide psychological or spiritual support to enhance quality of life before death, and foster death with dignity is a more meaningful and proper therapeutic option. ${ }^{34}$ Even if such patients survive the index admission, end-of-life care or advance care planning could be continued in the community by "community geriatric assessment teams" who support people in residential care homes and "integrated community care services" for patients living in their own homes in the community.

\section{Discharge planning}

Because of increasing vulnerability to stress with advancing age, not only pre-frail and frail people but also robust older people are likely to experience a decline in physical and mental wellbeing after hospitalisation for an acute illness. ${ }^{17,35,36}$ The resultant increase in frailty is associated with increasing risk of subsequent hospitalisation, ${ }^{36}$ which in turn predisposes patients to become more frail with repeated admission, forming a vicious cycle that ultimately terminates in death. Given that frailty is a dynamic, modifiable process, ${ }^{5,6}$ early intervention during the post-discharge period may revert patients back to their premorbid state and prevent hospitalisation.

Frailty assessment before discharge and comparison with the pre-admission state facilitate recognition of change in physical activity and function in individual patients. Then, targeted interventions for frailty can be planned. Exercise training and nutritional supplementation are the main components of such interventions. Exercise programmes with emphasis on resistance training have been shown to improve physical functioning and reduce frailty, ${ }^{37-40}$ and the effects are more prominent for moderately frail patients than for severely frail patients. ${ }^{37}$ Exercise with concomitant nutritional supplementation to augment caloric intake can further improve muscle strength. ${ }^{38}$ Cognitive training has also been shown to be effective. $^{39,40}$ Further, combined interventions (comprising exercise, nutrition, and cognitive training) have additional advantages over individual therapies in reducing frailty. ${ }^{39,40}$

There has been a paucity of study to determine which level of frailty can derive the most benefits from targeted interventions. However, considering that most studies were performed in frail people who were ambulatory and without severe cognitive impairment, patients at less severe levels of frailty are more likely to benefit from such interventions. Nevertheless, performing a pre-discharge CGA to guide post-discharge management plans is justified. For patients with less severe frailty, especially those who had good premorbid functional states, referral to geriatric day hospitals for targeted intervention is warranted. For severely frail people with limited ambulation, therapy to prevent complications resulting from the functional decline, such as supporting their nutritional state and preventing pressure sores or contractures, is more appropriate. For very severely frail patients with limited life expectancy, end-of-life programmes should be continued after discharge. As an integrated part of management, patients' health states and care plans should be re-evaluated at regular intervals.

\section{Present challenges}

International guidelines have recommended assessment for frailty during management of older 
people to facilitate the provision of integrated goaloriented care. ${ }^{1,9}$ However, frailty has not yet been formally introduced into clinical practice guidelines in Hong Kong. A standardised approach for frailty is essential, which should start with identification of people with frailty. Selection of an easy-to-use screening tool is required. The FRAIL scale, which only requires answers to five simple questions and has been studied in the Hong Kong population, seems to be a promising screening tool. ${ }^{41}$ Similarly, single-item measurements of walking speed or grip strength have also been shown to be suitable. ${ }^{42}$ In the United Kingdom, an electronic frailty index that uses electronic health record data was developed for use in clinical practice to identify and stratify frailty in older patients. ${ }^{43}$ In Hong Kong, a similar concept was used by the Hospital Authority to generate HARRPE (Hospital Admission Risk Reduction Programme for the Elderly) scores that identify patients at high risk of readmission. ${ }^{44}$ Thus, it is possible to generate frailty scores by using existing electronic health record data to alert us to people who are frail, so that targeted interventions and appropriate management plans can be offered. The subsequent pathway (which should differ according to frailty level) and setting (in hospital or community) for care upon detection of frailty also needs to be determined.

Frailty involves multiple organ systems and affects health outcomes. Its management is complicated by underlying co-morbidities and functional and cognitive decline. Therefore, the management approach should shift from the conventional disease-based approach towards an integrated goal-oriented approach, guided by multidisciplinary CGA. Frailty is an issue for not only geriatricians but also all health care workers who provide care to older people. Thus, consideration of frailty throughout the health care sector is required. Finally, studies showing the cost-effectiveness of the implementation of frailty assessments into care for older people are expected, as they might convince the policy maker to incorporate frailty assessment into health care system in the long run.

\section{Conclusion}

Frailty is associated with a range of adverse health outcomes. Incorporating the concept of frailty into clinical practice, which should involve multidisciplinary CGA, could enable better risk stratification and prediction of health outcomes. This would also facilitate a change from the conventional disease-based approach to a "whole patient" goaloriented approach. Further, it is possible to revert or reduce frailty through targeted intervention, so that early identification with appropriate management could maintain older people's independence in the community. Although there are challenges regarding the implementation of frailty assessments into the
Hong Kong health care system, it is time to take the initiative to consider frailty during daily practice and to accumulate experience so that a full-blown care pathway for frailty management can be developed.

\section{Author contributions}

The author has contributed to the concept or design, acquisition of data, analysis or interpretation of data, drafting of the article, and critical revision for important intellectual content.

\section{Declaration}

The author has disclosed no conflicts of interest. The author had full access to the data, contributed to the study, approved the final version for publication, and takes responsibility for its accuracy and integrity.

\section{References}

1. Morley JE, Vellas B, van Kan GA, et al. Frailty consensus: a call to action. J Am Med Dir Assoc 2013;14:392-7.

2. Fried LP, Ferrucci L, Darer J, Williamson JD, Anderson G. Untangling the concepts of disability, frailty, and comorbidity: implications for improved targeting and care. J Gerontol A Biol Sci Med Sci 2004;59:255-63.

3. Fried LP, Tangen CM, Walston J, et al. Frailty in older adults: evidence for a phenotype. J Gerontol A Biol Sci Med Sci 2001;56:M146-56.

4. Collard RM, Boter H, Schoevers RA, Oude Voshaar RC. Prevalence of frailty in community-dwelling older persons: a systematic review. J Am Geriatr Soc 2012;60:1487-92.

5. Lee JS, Auyeung TW, Leung J, Kwok T, Woo J. Transitions in frailty states among community-living older adults and their associated factors. J Am Med Dir Assoc 2014;15:2816.

6. Gill TM, Gahbauer EA, Allore HG, Han L. Transitions between frailty states among community-living older persons. Arch Intern Med 2006;166:418-23.

7. Mitnitski AB, Mogilner AJ, Rockwood K. Accumulation of deficits as a proxy measure of aging. Scientific World Journal 2001;1:323-36.

8. Song X, Mitnitski A, Rockwood K. Prevalence and 10-year outcomes of frailty in older adults in relation to deficit accumulation. J Am Geriatr Soc 2010;58:681-7.

9. Turner G, Clegg A, British Geriatrics Society, Age UK, Royal College of General Practitioners. Best practice guidelines for management of frailty: a British Geriatric Society, Age UK and Royal College of General Practitioners report. Age Ageing 2014;43:744-7.

10. Morley JE, Malmstrom TK, Miller DK. A simple frailty questionnaire (FRAIL) predicts outcomes in middle aged African Americans. J Nutr Health Aging 2012;16:601-8.

11. Rolfson DB, Majumdar SR, Tsuyuki RT, Tahir A, Rockwood K. Validity and reliability of the Edmonton Frail Scale. Age Aging 2006;35:526-9.

12. Rockwood K, Song X, MacKnight C, et al. A global clinical measure of fitness and frailty in elderly people. CMAJ 2005;173:489-95.

13. Geriatric Medicine Research, Faculty of Medicine, Dalhousie University. Research / projects, Clinical Frailty Scale@. Available from: http://geriatricresearch.medicine. dal.ca/clinical_frailty_scale.htm. Accessed 27 Oct 2018.

14. Woo J, Leung J, Morley JE. Comparison of frailty indicators 
based on clinical phenotype and the multiple deficit approach in predicting mortality and physical limitation. J Am Geriatr Soc 2012;60:1478-86.

15. Malmstrom TK, Miller DK, Morley JE. A comparison of four frailty models. J Am Geriatr Soc 2014;62:721-6.

16. Rubenstein LZ, Struck AE, Siu AL, Wieland D. Impacts of geriatric evaluation and management programs on defined outcomes: overview of the evidence. J Am Geriatr Soc 1991;39(9 Pt 2):8S-16S.

17. Clegg A, Young J, Iliffe S, Rikkert MO, Rockwood K. Frailty in elderly people. Lancet 2013;381:752-62.

18. Ellis G, Whitehead MA, O'Neill D, Langhorne P, Robinson D. Comprehensive geriatric assessment for older adults admitted to hospital. Cochrane Database Syst Rev 2011(7):CD006211.

19. Hospital Authority, Hong Kong SAR Government. Hospital Authority Statistical Report 2016-2017. Available from: http://www.ha.org.hk/haho/ho/stat/HASR16_17. pdf. Accessed 27 Oct 2018.

20. Jørgensen R, Brabrand M. Screening of the frail patient in the emergency department : a systematic review. Eur J Intern Med 2017;45:71-3.

21. Salvi F, Morichi V, Grilli A, et al. Screening for frailty in elderly emergency department patients by using the Identification of Seniors At Risk (ISAR). J Nutr Health Aging 2012;16:313-8.

22. Hastings SN, Purser JL, Johnson KS, Sloane RJ, Whitson HE. Frailty predicts some but not all adverse outcomes in older adults discharged from the emergency department. J Am Geriatr Soc 2008;56:1651-7.

23. Sirois MJ, Griffith L, Perry J, et al. Measuring frailty can help emergency departments identify independent seniors at risk of functional decline after minor injuries. J Gerontol A Biol Sci Med Sci 2017;72:68-74.

24. Robinson TN, Walston JD, Brummel NE, et al. Frailty for surgeons: review of a National Institute on Aging Conference on frailty for specialists. J Am Coll Surg 2015;221:1083-92.

25. Makary MA, Segev DL, Pronovost PJ, et al. Frailty as a predictor of surgical outcomes in older patients. J Am Coll Surg 2010;210:901-8.

26. Baztán JJ, Suárez-García FM, López-Arrieta J, RodríguezMañas L, Rodríuez-Artalejo F. Effectiveness of acute geriatric units on functional decline, living at home, and case fatality among older patients admitted to hospital for acute medical disorders: meta-analysis. BMJ 2009;338:b50.

27. Ekerstad N, Karlson BW, Dahlin Ivanoff S, et al. Is the acute care of frail elderly patients in a comprehensive geriatric assessment unit superior to conventional acute medical care? Clin Intev Aging 2016;12:1-9.

28. Ekerstad N, Dahlin Ivanoff S, Landahl S, et al. Acute care of severely frail elderly patients in a CGA-unit is associated with less functional decline than conventional acute care. Clin Interv Aging 2017;12:1239-49.

29. Kulmala J, NyKänen I, Hartikainen S. Frailty as a predictor of all-cause mortality in older men and women. Geriatr Gerontol Int 2014;14:899-905.

30. Kulminski AM, Ukraintseva SV, Kulminskaya IV, Arbeev KG, Land K, Yashin AI. Cumulative deficits better characterize susceptibility to death in elderly people than phenotypic frailty: lessons from the Cardiovascular Healthy Study. J Am Geriatr Soc 2008;56:898-903.

31. Dupre ME, Gu D, Warner D, Yi Z. Frailty and type of death among older adults in China: prospective cohort study. BMJ 2009;338:b1175.

32. Rockwood K, Rockwood MR, Mitnitski A. Physiological redundancy in older adults in relation to the change with age in the slope of a frailty index. J Am Geriatr Soc 2010;58:318-23.

33. Wallis SJ, Wall J, Biram RW, Romero-Ortuno R. Associations of the clinical frailty scale with hospital outcomes. QJM 2015;108:943-9.

34. Luk JK. End-of-life services for older people in residential care homes in Hong Kong. Hong Kong Med J 2018;24;63-7.

35. Gill TM, Gahbauer EA, Han L, Allore HG. The relationship between intervening hospitalizations and transitions between frailty states. J Gerontol A Biol Sci Med Sci 2011;66:1238-43.

36. Kahlon S, Pederson J, Majumdar SR, et al. Association between frailty and 30-day outcomes after discharge from hospital. CMAJ 2015;187:799-804.

37. Gill TM, Baker DI, Gottschalk M, Peduzzi PN, Allore $\mathrm{H}$, Byers A. A program to prevent functional decline in physically frail, elderly persons who live at home. N Engl J Med 2002;347:1068-74.

38. Fiatarone MA, O'Neill EF, Ryan ND, et al. Exercise training and nutritional supplementation for physical frailty in very elderly people. N Engl J Med 1994;330:1769-75.

39. Puts MT, Toubasi S, Andrew MK, et al. Interventions to prevent or reduce the level of frailty in communitydwelling older adults: a scoping review of the literature and international policies. Age Ageing 2017;46:383-92.

40. Ng TP, Feng L, Nyunt MS, et al. Nutritional, physical, cognitive, and combination interventions and frailty reversal among older adults: a randomized controlled trial. Am J Med 2015;128:1225-36.

41. Woo J, Yu R, Wong M, Yeung F, Wong M, Lum C. Frailty screening in the community using FRAIL scale. J Am Med Dir Assoc 2015;16:412-9.

42. Auyeung TW, Lee JS, Leung J, Kwok T, Woo J. The selection of a screening test for frailty identification in communitydwelling older adults. J Nutr Health Aging 2014;18:199203.

43. Clegg A, Bates C, Young J, et al. Development and validation of an electronic frailty index using routine primary care electronic health record data. Age Ageing 2016;45:353-60.

44. Chan S, Kwong P, Kong B, et al. Improving health of high risk elderly in the community-the HARRPE. Available from: www.healthyhkec.org/SCE/SCE3/abstracts/p37.pdf. Accessed 27 Oct 2018. 